\title{
Educación física y universidad: Evaluación de una experiencia docente a través del aprendizaje cooperativo \\ Physical Education and university: Evaluation of a teaching experience through cooperative learning \\ *José Manuel Bermejo Díaz, *David Pulido Salas, *Aina Maria Galmes-Panades, ***Pedrona Serra Payeras, *Josep Vidal Conti, *Francisco Javier Ponseti Verdaguer* \\ *Universidad de Las Islas Baleares (España), ** Universidad de Barcelona (España)
}

Resumen. El aprendizaje cooperativo es una metodología educativa basada en la interacción en grupos de trabajo reducidos, en los que el alumnado interioriza la corresponsabilidad en el aprendizaje de todo tipo de contenidos. En los últimos años esta metodología inclusiva se está instaurando en las aulas de educación infantil, primaria y secundaria de las Islas Baleares. Por ello, este trabajo nace de la implementación de una experiencia docente universitaria a través del aprendizaje cooperativo en la asignatura de segundo curso del grado de educación primaria titulada «Educación Física y su didáctica» situada en el departamento de Pedagogía y Didácticas específicas de la Universidad de las Islas Baleares. El objetivo de este trabajo es determinar el grado de aceptación del alumnado de la asignatura de Educación física y su didáctica basándose en la utilización del trabajo cooperativo. Destaca la utilización del diario de prácticas como herramienta para el desarrollo de la asignatura. Los resultados obtenidos son satisfactorios en cuanto a la valoración de la asignatura por parte del alumnado, a través del aprendizaje cooperativo, en variables como satisfacción, rendimiento, implicación del alumnado y evaluación del profesorado, pues su puntuación es superior a 8 sobre 10. Del mismo modo, la puntuación obtenida en los roles de aprendizaje cooperativo es de 8 sobre 10. Con la obtención de los resultados se confirma la positiva aceptación por parte del alumnado de la metodología del aprendizaje cooperativo como recurso en el grado de educación primaria y, consecuentemente, se propone la implementación de la misma metodología como contenido.

Palabras Clave: Educación Física, aprendizaje cooperativo, universidad, proceso enseñanza-aprendizaje.

\begin{abstract}
Cooperative learning is an educational methodology based on the interaction between work groups of 3-5 members. These members share the responsibility in their learning. This methodology is increasingly prevalent in pre-school education and primary and secondary education in Balearic Islands. For that reason, the present study was developed from the implementation of a teaching experience throughout cooperative learning in the subject of Physical Education during the second year of the primary education degree in the Department of Pedagogy and specific Didactics at the University of the Balearic Islands. The aim is to determine pupils' degree of acceptance of the Physical Education subject based on cooperative learning. An important highlight lays in the use of practice logs as a main tool used for the development of the subject. Very satisfactory and promising results are shown regarding the assessment of the subject through cooperative learning in variables such as satisfaction, performance, students' involvement and teacher evaluation, with a score higher than 8 over 10 . According to the present results, students' positive acceptance of the cooperative learning methodology in the primary education degree is confirmed. We propose to include cooperative learning also as a content of the subject, apart from as a learning methodology.
\end{abstract}

Keywords: Physical Education, cooperative learning, university, teaching-learning process.

\section{Introducción}

En la última década se han puesto en marcha abundantes experiencias de innovación docente con el objetivo de mejorar el proceso de enseñanza-aprendizaje del alumnado universitario, de entre las que destaca la implementación del aprendizaje cooperativo en la educación física (Hernández, 2018).

El aprendizaje cooperativo es una metodología educativa basada en la interacción en grupos de trabajo reducidos, generalmente heterogéneos, en los que el alumnado, a diferencia del trabajo grupal, interioriza la corresponsabilidad en el aprendizaje de todo tipo de contenidos. Este tipo de aprendizaje exige al alumnado la toma de conciencia sobre los contenidos a tratar, la estructuración cognitiva y la toma de decisiones para poder establecer la comunicación intragrupal. La educación física cumple las condiciones idóneas para fomentar la educación en valores y, frecuentemente, se trabaja a través de la metodología cooperativa (Velázquez, 2015).

Fecha recepción: 08-02-20. Fecha de aceptación: 19-05-20

Aina Maria Galmés Panadés

aina.galmes.panades@gmail.com
Si bien, para que se produzca el aprendizaje cooperativo es imprescindible que el grupo clase se encuentra bajo un clima de tolerancia, respeto y empatía (Pérez-Pueyo y HortigüelaAlcalá, 2019).

Según Johnson y Johnson (1999) identifican cinco condicionantes indispensables para promover el aprendizaje cooperativo: (1) interdependencia positiva de metas, así como de recursos y de roles; (2) interacción promotora, o manifestación de conductas de ayuda, apoyo y ánimo dentro del grupo; (3) responsabilidad individual; (4) habilidades interpersonales y de trabajo en pequeño grupo, y (5) procesamiento o autoevaluación grupal, centrada en propiciar la identificación de las conductas manifestadas durante el trabajo grupal por parte de todos los componentes del equipo cooperativo.

Las sucesivas y cambiantes leyes (LOGSE, LOE, LOMCE) del sistema educativo llevan más de 25 años situando al aprendizaje cooperativo como el eje de nuestro sistema educativo (Fernández-Río y Méndez-Giménez, 2016). En la actualidad, es apreciable el notable auge del concepto «cooperación» (Fernández-Río, 2017). De este modo, el aprendizaje cooperativo es considerado un modelo de enseñanza existente en educación física que abarca un planteamiento glo- 
bal del proceso de enseñanza-aprendizaje (Fernández-Río y Méndez-Giménez, 2016).

La gran diferencia de niveles motrices y de condición física son notables en las aulas universitarias, y hace que el alumnado con menos experiencias motrices, habilidades o condición física se desmotive. El trabajo cooperativo minimiza estas desigualdades, ofrece posibilidades ajustadas para todos en función de sus capacidades, conlleva una organización de las actividades académicas en grupos de trabajo reducidos y heterogéneos, así como una reestructuración del proceso de enseñanza-aprendizaje que hace variar la relación alumnado-profesorado y alumnado-alumnado, y supone un modelo de co-aprendizaje entre alumnos, alumnas y docentes en el que el grupo cooperativo trabaja por alcanzar un objetivo común (Izquierdo, Asensio, Escarbajal y Rodríguez, 2019).

Asimismo, Casey y Goodyear (2015) demuestran que este planteamiento didáctico desarrolla aprendizajes de carácter físico, cognitivo, social y afectivo. Trabajos como el de Lamoneda, González-Villora y Fernández-Río (2020) han valorado con éxito la implementación del aprendizaje cooperativo en educación física (Casey y Goodyear, 2015; FernándezRío, 2014; Gillies, 2014).

Además, la eficacia del aprendizaje cooperativo para promover, tanto el aprendizaje motor como el desarrollo de conductas prosociales se ha investigado recientemente (Johnson y Johnson, 2014; Lavega, Planas y Ruiz, 2014; Velázquez, Fraile y López-Pastor, 2014), fomentando la inclusión del alumnado con necesidades específicas de apoyo educativo (Armada, Gonzálezy Montávez, 2013; Cherng, Turney y Kao, 2014; Latorre-Cosculluela, Liesa-Orús y Vázquez-Toledo, 2018), para la mejora del rendimiento académico (Martín, 2014; Pelaz, 2018), aumentar la motivación en el alumnado (Herrada y Baños, 2018; Prieto, 2018) y la mejora del autoconcepto (Navarro-Patón, Rego y García, 2018).

En la misma línea, la aplicabilidad del trabajo cooperativo ha sido importante en diferentes contenidos de la educación física: habilidades básicas (Fernández-Río, 2010); habilidades gimnásticas (Bayraktar, 2011; Fernández-Río y MéndezGiménez, 2013); condición física y salud (Hannon y Ratlifee, 2004); actividades rítmicas (Crespo, 2014; Ramos y Hernangómez, 2014) y actividades en el medio natural (Cueto, 2014).

De este modo, el trabajo cooperativo se postula como una competencia fundamental para el futuro profesorado, que favorece que el alumnado comparta unas metas y valores de forma cohesionada y participativa (Izquierdo et al., 2019). Además, se ha demostrado un interés creciente en la formación del profesorado de educación física para la mejora de sus habilidades, la autoeficacia y el conocimiento pedagógico (Legrain, Escalié, Lafont y Chaliès, 2019).

Según Hernández (2018) llevar a cabo los trabajos prácticos grupales en la educación física universitaria hace que surjan problemáticas, y por ello implementa una experiencia en su asignatura basada en el trabajo cooperativo. No es tarea fácil implementar una nueva experiencia docente centrada en el trabajo cooperativo en la educación física universitaria debido a que el alumnado no está acostumbrado a trabajar desde esta perspectiva.

Por todo ello, el objetivo del presente estudio es determi- nar el grado de aceptación del alumnado de la asignatura de Educación física y su didáctica en la Universidad de las Islas Baleares en base a la utilización del trabajo cooperativo, a través de herramientas como el diario de prácticas.

\section{Material y métodos}

\section{Contexto y participantes}

En la asignatura de Educación física y su didáctica, formada por cuatro grupos-clase, se desarrolló el proyecto de innovación docente titulado «Aprender a cooperar en el aula de educación física: Juntos aprendemos más», en la Universidad de las Islas Baleares. Cada grupo-clase tenía horarios y profesorado distinto, pero todos los grupos compartían contenidos y se regían por la misma guía docente. Para garantizar la adecuada coordinación entre grupos, el profesorado de los cuatro grupos realizó 5 reuniones antes de empezar la asignatura, 2 reuniones durante la impartición de la misma, y 3 reuniones al terminar la asignatura. Las instrucciones para la realización del trabajo fueron las mismas para todos los grupos, así como la metodología de trabajo cooperativo.

La muestra estuvo constituida por 156 alumnos y alumnas de segundo curso, de la Universidad de las Islas Baleares. La muestra total estaba compuesta por 109 mujeres (69.9\%) y 47 hombres (30.1\%). El grupo 1 estaba formado por un total de 40 estudiantes, 24 mujeres $(60 \%)$ y 16 hombres (40\%). El grupo 2 estaba formado por un total de 34 estudiantes, 26 mujeres $(76.5 \%)$ y 8 hombres $(23.5 \%)$. El grupo 3 estaba formado por un total de 54 estudiantes, 42 mujeres $(77.8 \%)$ y 12 hombres (22.2\%). Y el grupo 4 estaba formado por un total de 28 estudiantes, 17 mujeres $(60.7 \%)$ y 11 hombres (39.3\%) (Ver tabla 1). El alumnado tenía edades comprendidas entre los 19 y los 40 años. El alumnado participante residía en Mallorca (Islas Baleares), España. Como criterio de inclusión el alumnado debía estar matriculado en la asignatura de Educación física y su didáctica, en el curso académico 2018-19. Fue criterio de exclusión no realizar el trabajo del diario de prácticas en grupo.

\begin{tabular}{|c|c|c|c|}
\hline \multirow[t]{2}{*}{ Grupos } & \multirow[b]{2}{*}{$\mathrm{n}$ total } & \multicolumn{2}{|c|}{ Distribución por sexo } \\
\hline & & Mujeres n, (\%) & Hombres n, (\%) \\
\hline Grupo 1 & 40 & $24(60 \%)$ & $16(40 \%)$ \\
\hline Grupo 2 & 34 & $26(76.5 \%)$ & $8(23.5 \%)$ \\
\hline Grupo 3 & 54 & $42(77.8 \%)$ & $12(22.2 \%)$ \\
\hline Grupo 4 & 28 & $17(60.7 \%)$ & $11(39.3 \%)$ \\
\hline Conjunto de los 4 grupos & 156 & $109(69.9 \%)$ & $47(30.1 \%)$ \\
\hline
\end{tabular}

\section{Procedimiento}

Para la realización de este estudio se informó previamente a los y las participantes de las características del trabajo cooperativo y su relación con la asignatura. La información se obtuvo a partir del diario de prácticas. El diario de prácticas se realizó sobre los contenidos trabajados en las sesiones prácticas y se entregó en dos partes, una entrega a mitad de cuatrimestre con la primera mitad de las sesiones, y otra entrega al finalizar la asignatura con la segunda mitad de las sesiones.

Todo el diario debía realizarse a través de la metodología de trabajo cooperativo. Se hicieron grupos estables o «base» (Pujolás, 2008b) para todo el curso, formados por 4 alumnos $\mathrm{y}$ alumnas cada uno. Algunos grupos tuvieron una compo- 
sición de entre 3 y 6 personas debido a las altas y las bajas en la asignatura. Los grupos los hizo el alumnado de manera autónoma, pero se les explico que para favorecer el trabajo cooperativo debían ser grupos heterogéneos. Siguiendo el ciclo de aprendizaje cooperativo de Fernández-Río (2017), la fase 1, de creación y cohesión de grupo, se llevó a cabo la primera semana de curso, especialmente en las sesiones prácticas, en las que se realizaron dinámicas con diferentes agrupaciones para favorecer la relación entre el alumnado, facilitar la desinhibición y crear un clima de confianza entre el grupo-clase. La fase 2, orientada a aprender a usar el aprendizaje cooperativo para aprender se trabajó en las sesiones teóricas y en las prácticas. En las sesiones prácticas se fomentó la cooperación en lugar de la competición y el logro colectivo a través de actividades variadas. En las sesiones teóricas se realizaban actividades por grupos de trabajo dirigidas a la mejora de los diarios y a la resolución de dudas, además de trabajar contenidos teóricos a través de estructuras cooperativas, como: el folio giratorio, 1-2-4 o lápiz al centro (Naranjo y Lago, 2017). Por último, la fase 3, el aprendizaje cooperativo como recurso para enseñar y aprender, se trabajó con la realización del diario de prácticas, a través del cual el alumnado se veían forzados a cooperar de manera regular, partiendo de experiencias previas de trabajo en contextos cooperativos en las sesiones teóricas y prácticas de la asignatura.

Como parte de la metodología de aprendizaje cooperativo, se indicó al alumnado que debía repartir unos roles dentro de los grupos de trabajo, para favorecer la organización y la participación (Naranjo y Lago, 2017; Pujolàs et al., 2014). El objetivo de establecer roles fue que los y las participantes se responsabilizaran de un aspecto del trabajo, para facilitar la coordinación entre las personas del grupo y favorecer el trabajo cooperativo, siendo así responsables últimos del trabajo (Tabla 2). Cabe destacar que, aunque se establecieron roles definidos, cada persona del grupo debía revisar el trabajo realizado por los demás componentes del grupo, convirtiéndose así en responsable de todos los aspectos del trabajo, cumpliendo así con una de las características del trabajo cooperativo, la interdependencia positiva.

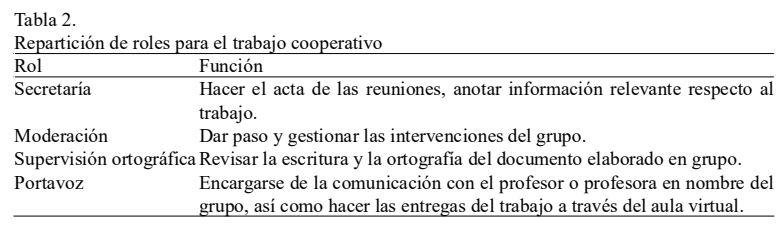

En cuanto a la evaluación de la asignatura Educación física y su didáctica, el diario de prácticas tenía un peso del $30 \%$ sobre la nota total de la asignatura, que se estructuraba desde la relación teórico-práctica con base cooperativa.

\section{Descripción de instrumentos}

El diario de prácticas estaba compuesto por cuatro partes, las tres primeras hacían referencia a cada sesión, y la cuarta hacía referencia al conjunto del diario. La primera parte estaba formada por un conjunto de 3 preguntas abiertas; la segunda por una evaluación del alumnado sobre la sesión; en la tercera parte se les encargaba diseñar actividades relacionadas con los contenidos de la sesión, y la cuarta parte consistía en una autoevaluación sobre el trabajo cooperativo de su propio grupo. De cada sesión práctica, el alumnado debía responder de manera consensuada a tres preguntas. Se establecieron los ítems de aprendizaje referentes a cada pregunta, de cada una de las sesiones, y a partir de dichos ítems se evaluó el aprendizaje en 3 niveles: cognitivo, afectivo, y emocional. Las preguntas eran las siguientes: a) ¿qué hemos aprendido?; buscando relación con los contenidos de la asignatura y otros aprendizajes como el trabajo en equipo, la creatividad, la desinhibición, etc.; b) ¿cómo nos hemos sentido durante la sesión?, buscando una reflexión personal sobre la cohesión de grupo, la educación emocional y la relación entre compañeros y compañeros y docente; y, c) ¿cómo relacionas la sesión práctica con los contenidos teóricos de la asignatura? (Anexo 1). Referente a la segunda parte del diario, en cada sesión el alumnado debía evaluar la efectividad del aprendizaje cooperativo para el aprendizaje, a través de varios ítems, de acuerdo con (Naranjo y Lago, 2017; Pujolàs et al., 2014; Woods y Chen, 2011). Los ítems utilizados para evaluar la efectividad del aprendizaje cooperativo para el aprendizaje fueron: bienestar, grado de participación, clima de clase, comunicación alumnado-alumnado, alumnado-profesorado, profesorado-alumnado; consecución de objetivos, el trabajo individual, el trabajo del grupo y el trabajo del profesor o profesora (Tabla 3 y Anexo 2). Referente a la tercera parte del diario, después de cada sesión se pedía al grupo que diseñaran actividades relacionadas con los contenidos de la sesión, dando algunas premisas como: qué contenidos debían tratar, metodologías específicas a utilizar, para qué curso o ciclo, o para qué parte de la sesión debían diseñar las actividades (Anexo 3). Y, por último, referente a la cuarta parte del diario, el alumnado realizó una evaluación del trabajo cooperativo realizado por su grupo de trabajo al finalizar la asignatura (Anexo 4). En ambas entregas se facilitó un feedback muy detallado, teniendo tutorías de una hora con cada grupo realizando una evaluación intermedia que facilitó la comprensión de muchos aspectos que en un inicio el alumnado no había entendido, y mejoró la calidad de los trabajos en la segunda entrega. Para la corrección de ambas entregas se utilizó una rúbrica común para los cuatro de la asignatura (Anexo 5).

\begin{tabular}{ll}
$\begin{array}{l}\text { Tabla 3. } \\
\text { Definición de los constructos utilizados para la valoración de las sesiones. }\end{array}$ \\
\hline \multicolumn{1}{c}{ Constructo } & \multicolumn{1}{c}{ Definición } \\
\hline Bienestar & Satisfacción y tranquilidad fisica y mental durante la sesión \\
\hline Participación & $\begin{array}{l}\text { Intervención voluntaria y significativa en las diversas } \\
\text { actividades de la sesión }\end{array}$ \\
\hline Clima & $\begin{array}{l}\text { Situación ambiental del aula que favorece el proceso de } \\
\text { enseñanza-aprendizaje }\end{array}$ \\
\hline Comunicación & $\begin{array}{l}\text { Comunicar de manera eficiente, útil y agradable diversos } \\
\text { aspectos relacionados con la sesión, entre iguales de manera } \\
\text { bidireccional y entre profesorado y alumnado, también de } \\
\text { manera bidireccional. }\end{array}$ \\
\hline Consecución de objetivos & Lograr alcanzar los objetivos propuestos para la sesión. \\
\hline Trabajo alumnado & $\begin{array}{l}\text { Participar con interés, uno mismo, a lo largo de toda la sesión } \\
\text { e involucrarse en las actividades para favorecer el proceso de } \\
\text { enseñanza-aprendizaje. }\end{array}$ \\
\hline Trabajo compañeros/as & $\begin{array}{l}\text { Participar, las personas del grupo, con interés a lo largo de } \\
\text { toda la sesión e involucrarse en las actividades para favorecer } \\
\text { el proceso de enseñanza-aprendizaje. }\end{array}$ \\
\hline Trabajo profesor/a & $\begin{array}{l}\text { Gestionar el desarrollo de las actividades a lo largo de la } \\
\text { sesión para fomentar la participación e involucración del } \\
\text { alumnado en las actividades para favorecer el proceso de } \\
\text { enseñanza-aprendizaje. }\end{array}$ \\
\hline
\end{tabular}

También se utilizaron las encuestas de evaluación docente realizadas por la universidad para conocer la nota media otorgada por el alumnado al profesorado de la Universidad, del departamento y de la asignatura. 


\section{Análisis de datos}

Para la valoración de las sesiones prácticas se realizó la media y la desviación estándar (DE) de las puntuaciones otorgadas por el alumnado en cada sesión, de los siguientes ítems: participación, clima de clase, comunicación (alumnadoalumnado, alumnado-profesorado, profesorado-alumnado), consecución de objetivos, trabajo individual, trabajo de grupo, trabajo del profesor o profesora. Los datos se analizaron realizado un análisis ANOVA(Tabla 4).

Como análisis de sensibilidad se ha realizado el mismo análisis que en la tabla 4, haciendo una diferenciación entre la primera entrega del diario referida a las 6 primeras sesiones, y la segunda entrega del diario, referida a las 6 últimas sesiones (Tabla 5).

Para la valoración global de cada sesión se realizó la media y la DE de la puntuación otorgada por el alumnado en cada uno de los siguientes aspectos: Bienestar, participación, clima, comunicación (alumnado-alumnado, alumnadoprofesorado, profesorado-alumnado) y consecución de objetivos. Para analizar esta información se realizó un análisis ANOVA(Tabla 6).

Como parte de la valoración del trabajo cooperativo, al finalizar la asignatura, cada grupo de trabajo debía auto-evaluar cómo habían trabajado de manera cooperativa, evaluando los siguientes ítems: a) rol (secretaría, moderación, supervisión ortográfica y portavoz), b) tareas, referido a la repartición equitativa de las mismas entre el grupo de trabajo; c) reuniones, referido a si habían organizado, asistido y participado en las reuniones de manera cooperativa como grupo; d) reparto equitativo del trabajo, en relación a tareas, responsabilidades, rol, participación, debates, etc.; e) consenso, referido a las habilidades del grupo para llegar a un consenso grupal, a través del trabajo cooperativo; y f) debate, en relación a la capacidad de generar un debate que favorezca el aprendizaje y el crecimiento del grupo. Se realizó un análisis ANOVA. Como elemento informativo se incluye en la tabla la puntuación obtenida por cada grupo en la cualificación del diario de prácticas (Tabla 7).

Todos los análisis se han realizado con el programa estadístico Stata versión 15.1.

\section{Resultados}

\section{Nota media del profesorado}

A través de las encuestas anónimas de satisfacción del alumnado, se obtuvieron los siguientes resultados: la nota media del profesorado de la universidad es de 7.83 puntos en una escala de 10. La nota media del profesorado del departamento de pedagogía y didácticas específicas es de 7.61. Y la nota media del profesorado que ha impartido la asignatura de Educación física y su didáctica es de 9.35 puntos. El objetivo del presente estudio fue determinar el grado de aceptación del alumnado en base a la utilización del trabajo cooperativo, y a través de herramientas como el diario de prácticas. A pesar de que la información presentada no responde de manera directa al objetivo, si lo hace de manera indirecta, ya que muestra que la valoración que hace el alumnado sobre el profesorado, es mejor en esta asignatura que la valoración media de la universidad y del departamento.

\section{Valoración del alumnado: sesiones prácticas}

En la tabla 5 se presenta la media y la DE de la valoración realizada por el alumnado sobre las sesiones prácticas, en relación a los siguientes aspectos (Naranjo y Lago, 2017; Pujolàs et al., 2014): participación, clima, comunicación, consecución de objetivos, propio trabajo, trabajo de los compañeros, y trabajo del profesor o profesora. Todas las valoraciones tienen una nota superior a 8 puntos en una escala de 10 , siendo el valor mínimo 8.32 y el valor máximo 8.92 . Se encuentran diferencias significativas entre grupos de trabajo con relación a la valoración de la comunicación (alumnadoalumnado, alumnado-profesorado, profesorado-alumnado) y del trabajo del profesorado. A pesar de la acogida positiva de la implementación del trabajo cooperativo por parte del alumnado, las valoraciones de los diferentes aspectos de las sesiones en la segunda entrega del diario de prácticas fueron más bajas que las de la primera entrega. Este hecho puede deberse al cansancio acumulado del alumnado en la recta final del curso.

\begin{tabular}{|c|c|c|c|c|}
\hline Variables resultado & $\mathrm{N}$ total & Media & $\mathrm{DE}$ & Valor $\mathrm{p}$ \\
\hline Participación & 55 & 8.92 & 0.55 & 0.074 \\
\hline Clima & 54 & 8.83 & 0.62 & 0.271 \\
\hline Comunicación & 54 & 8.32 & 0.72 & 0.005 \\
\hline Consecución de objetivos & 53 & 8.84 & 0.61 & 0.603 \\
\hline Trabajo alumnado & 47 & 8.68 & 0.49 & 0.144 \\
\hline Trabajo compañeros/as & 47 & 8.77 & 0.47 & 0.724 \\
\hline Trabajo profesor/a & 47 & 8.71 & 0.73 & 0.015 \\
\hline
\end{tabular}

Los resultados expuestos corresponden a un análisis ANOVA, presentando los resultados en media y (DE). Estos valores corresponden a la valoración realizada por el alumnado sobre una escala de 10 puntos; es la media de todas las evaluaciones, una por cada sesión. La $n$ presentada corresponde a los grupos de trabajo, compuesto cada grupo por 3-6 personas.

En el anexo 1 se encuentran las preguntas del diario de prácticas, con las que se evaluó el aprendizaje a nivel cognitivo, afectivo y emocional. A pesar de no presentar resultados cualitativos sobre estas preguntas en el presente artículo, cabe destacar que el aprendizaje fue positivo en los tres niveles, pues las calificaciones del alumnado fueron de 7.5 puntos de media (sobre 10) en el diario de prácticas.

La tabla 5 presenta la media y la DE de la valoración realizada por el alumnado sobre las sesiones prácticas, pero en esta ocasión se compara la valoración realizada sobre las 6 primeras sesiones con la valoración realizada sobre las 6 segundas sesiones. Los aspectos valorados fueron: participación, clima, comunicación, consecución de objetivos, propio trabajo, trabajo de los compañeros y compañeras, y trabajo del profesor o profesora; haciendo diferenciación entre la primera y la segunda entrega del diario. Todas las valoraciones tienen una nota superior a 8 puntos en una escala de 10. Todas las valoraciones reciben una puntuación inferior en la segunda entrega, a excepción de la consecución de

\begin{tabular}{|c|c|c|c|c|c|c|c|}
\hline \multirow[b]{2}{*}{ Variables resultado } & \multicolumn{3}{|c|}{ Sesiones primera entrega } & \multicolumn{4}{|c|}{ Sesiones segunda entrega } \\
\hline & $\mathrm{N}$ total & Media & DE* & $\mathrm{N}$ total & Media & $\mathrm{DE}^{*}$ & Valor $\mathrm{p}$ \\
\hline Participación & 31 & 8.99 & 0.61 & 24 & 8.83 & 0.47 & 0.287 \\
\hline Clima & 30 & 8.92 & 0.68 & 24 & 8.72 & 0.55 & 0.260 \\
\hline Comunicación & 30 & 8.49 & 0.71 & 24 & 8.10 & 0.69 & 0.046 \\
\hline Consecución de objetivos & 29 & 8.83 & 0.62 & 24 & 8.85 & 0.62 & 0.936 \\
\hline Trabajo alumnado & 23 & 8.80 & 0.59 & 24 & 8.58 & 0.35 & 0.124 \\
\hline Trabajo compañeros/as & 23 & 8.83 & 0.58 & 24 & 8.71 & 0.33 & 0.394 \\
\hline Trabajo profesor/a & 23 & 8.93 & 0.59 & 24 & 8.49 & 0.79 & 0.039 \\
\hline
\end{tabular}


objetivos, que aumenta ligeramente. El mayor descenso se produce en la valoración de la comunicación y en el trabajo del profesor o profesora.

Los resultados expuestos corresponden a un análisis ANOVA, presentando los resultados en media y DE. Estos valores corresponden a la valoración realizada por el alumnado sobre una escala de 10 puntos; es la media de todas las evaluaciones, una por cada sesión. La $n$ presentada corresponde a los grupos de trabajo, compuesto cada grupo por 3-6 personas. El trabajo del diario cooperativo se entregó en dos partes. Cada parte estaba compuesta por 6 sesiones. En la presente tabla se hace una comparación de estas dos entregas.

En la tabla 6 se presentan los resultados de las valoraciones del alumnado sobre cada sesión con media y DE. Para hacer una valoración global de cada sesión se ha utilizado la valoración media de cada sesión, teniendo en cuenta los siguientes ítems: bienestar, participación, clima de clase, comunicación (alumnado-alumnado, alumnado-profesorado, profesorado-alumnado) y consecución de objetivos. Todas las sesiones fueron valoradas con notas superiores a 8 sobre 10 , siendo la puntuación mínima 8.10 y la puntación máxima 8.85 .

\begin{tabular}{|c|c|c|c|}
\hline Variables resultado & $\mathrm{N}$ total & Media & $\mathrm{DE}$ \\
\hline Sesión 1 & 32 & 8.83 & 0.67 \\
\hline Sesión 2 & 31 & 8.38 & 0.97 \\
\hline Sesión 3 & 32 & 8.81 & 0.69 \\
\hline Sesión 4 & 32 & 8.85 & 0.59 \\
\hline Sesión 5 & 32 & 8.82 & 0.69 \\
\hline Sesión 6 & 30 & 8.77 & 0.82 \\
\hline Sesión 7 & 31 & 8.59 & 0.76 \\
\hline Sesión 8 & 35 & 8.10 & 0.85 \\
\hline Sesión 9 & 35 & 8.38 & 0.73 \\
\hline Sesión 10 & 35 & 8.66 & 0.82 \\
\hline Sesión 11 & 35 & 8.75 & 0.55 \\
\hline Sesión 12 & 28 & 8.74 & 0.66 \\
\hline
\end{tabular}

Los resultados expuestos corresponden a un análisis ANOVA, presentando los resultados en media y DE. Estos valores corresponden a la valoración realizada por el alumnado sobre una escala de 10 puntos. Para la valoración global se ha tenido en cuenta la nota asignada a los siguientes aspectos: Bienestar, participación, clima de clase, comunicación ((alumnado-alumnado, alumnado-profesorado, profesorado-alumnado)) y consecución de objetivos. La $n$ presentada corresponde a los grupos de trabajo, compuesto cada grupo por 3-6 personas.

En el anexo 3 se presenta un ejemplo del tercer apartado del diario de prácticas, de cada una de las sesiones. A pesar de no presentar resultados sobre esta parte del diario, se considera oportuno explicar el objetivo de dicho apartado. Con estas preguntas se pretendía establecer una relación entre los contenidos teóricos y prácticos de la asignatura, para favorecer la capacidad de identificación y síntesis, la interconexión de conocimientos, el aprendizaje significativo, el liderazgo y la cooperación, entre otras capacidades y competencias, a través del aprendizaje cooperativo.

\section{Autoevaluación del trabajo cooperativo}

En la tabla 7 se presentan los resultados de la autoevaluación que realizaron los y las estudiantes al finalizar el trabajo del diario de prácticas. La autoevaluación constaba de la valoración de los siguientes ítems: rol, tareas, reuniones, reparto equitativo, consenso en las decisiones, debate, nota del grupo; siendo una autoevaluación global de todo el trabajo, es decir, al responder debían valorar todo el proceso de realización del diario de prácticas. Las valoraciones del alumnado fueron en todos los casos superiores a 3 puntos sobre una escala de 4 , siendo la puntuación mínima 3.40 y la máxima 3.60. Se encuentran diferencias significativas entre grupos de trabajo en relación a la valoración del reparto equitativo del trabajo y el consenso en la toma de decisiones. Como elemento informativo al final de la tabla se presenta la nota media del alumnado en la realización del trabajo del diario de prácticas.

\begin{tabular}{lcccc} 
Tabla 7. \\
\multicolumn{5}{l}{ Autoevaluación grupal del trabajo cooperativo sobre 4 puntos. } \\
\hline Variables resultado & $\mathrm{N}$ total & Media & $\mathrm{DE}$ & \\
Rol & 41 & 3.44 & 0.67 & 0.357 \\
Tareas & 42 & 3.57 & 0.55 & 0.717 \\
Reuniones & 42 & 3.60 & 0.54 & 0.190 \\
Reparto equitativo & 50 & 3.44 & 0.58 & 0.011 \\
Consenso en las decisiones & 50 & 3.54 & 0.58 & 0.004 \\
Debate & 48 & 3.40 & 0.68 & 0.753 \\
Nota del grupo sobre 10 puntos & 67 & 7.50 & 1.32 & 0.113 \\
\hline
\end{tabular}

Los resultados expuestos corresponden a un análisis ANOVA, presentando los resultados en media y DE. Estos resultados corresponden a una autoevaluación realizada el alumnado sobre una escala de 4 puntos. En grupos de cuatro personas los y las estudiantes debían distribuirse los siguientes roles: secretaria, moderación, supervisión ortográfica y portavoz. Debían repartirse las tareas de manera equitativa, programar y acudir a las reuniones para realizar el trabajo (un diario de prácticas), tomar decisiones de manera consensuada, y utilizar el debate como herramienta para construir conocimiento y consenso. La $n$ presentada corresponde a los grupos de trabajo, compuesto cada grupo por 3-6 personas. La nota del grupo corresponde a la nota obtenida por el grupo en el trabajo (diario de prácticas) sobre una escala de 10.

\section{Discusión}

El trabajo cooperativo en las aulas universitarias es de gran importancia no tan solo por la generación de conocimiento de los y las estudiantes, sino también por el aprendizaje humano que han propuesto durante toda la historia de la educación autores como Piaget a través del valor de la interacción social en el aprendizaje con la teoría del desarrollo cognitivo, o Vygotsky con su trabajo sobre la zona de desarrollo próximo (Maldonado, 2007). El aprendizaje cooperativo es considerado imprescindible para llevar a cabo una educación física de calidad, con un proceso crítico, reflexivo y constructivo en la educación primaria, por lo que se precisa una formación continuada de los y las actuales docentes y del actual estudiante de educación física en primaria (Cañabate Ortíz, D., Tesouro Cid., M., Puigggali Allepuz, J., y Zagalaz Sánchez, M., 2018).

En este artículo se analiza el grado de aceptación del alumnado de la asignatura de Educación física y su didáctica de los estudios de magisterio de primaria en la Universidad de las Islas Baleares. A continuación, se discute la información expuesta en el apartado anterior identificando los aspectos teóricos relevantes. En relación al objetivo planteado se ha concluido que el alumnado acoge muy positivamente esta metodología en la asignatura universitaria de Educación Física y su didáctica, por lo que se refiere a la asigna- 
ción de los roles del aprendizaje y en concreto al ítem de comunicación y de reunión para realizar los trabajos. El presente trabajo sigue la línea de Feria-Madueño, GrimaldiPuyana, Sánchez-Oliver y Alcaraz-Rodríguez (2017) que manifiestan que sus alumnos y alumnas del Grado en Educación Primaria con la mención de Educación Física valoran de forma muy positiva la implementación del aprendizaje cooperativo como oportunidad para conocer a los compañeros/as, desarrollar las competencias sociales y comprender mejor los contenidos.

Asimismo, el alumnado de la presente investigación valora positivamente los ítems relacionados con el entorno del proceso de enseñanza-aprendizaje, como son su bienestar durante las sesiones, la comunicación entre agentes, el clima del aula, la participación o la consecución de objetivos. Además, también han valorado positivamente la cooperación entre miembros del grupo para lograr el objetivo común, realizar el diario de prácticas. En la misma línea, Hernández (2018) destaca la gran satisfacción de su alumnado del Grado en Ciencias de la Actividad Física y el Deporte con la implementación del aprendizaje cooperativo en sus sesiones lo cual genera una mejora en la interacción y ambiente entre los compañeros y compañeras.

Así pues, gracias a la intervención se constata la acogida positiva por parte del alumnado con relación al trabajo cooperativo, como demuestra la valoración positiva del profesorado de la asignatura en comparación al resto de profesorado de la Universidad de las Islas Baleares. Los trabajos de Gibbons y Black (1997) y Fernández-Río (2003, 2017), demostraron que la educación física trabajada desde el aprendizaje cooperativo influye de manera muy positiva en la aceptación e interrelación social, la competencia física y la motivación por la asignatura. En la misma línea, Fernández-Río y Méndez-Giménez (2016), señalan que los profesores y profesoras que experimentaron el trabajo cooperativo no regresaron a sus clases expositivas; descubrieron que sus estudiantes aprendían más y mejor, que no abandonaban las clases, que se interesaban por la materia y que compartían con sus iguales elementos que iban más allá de las aulas.

Con relación a los resultados obtenidos en la autoevaluación del alumnado sobre el trabajo cooperativo (tabla 7), es frecuente en el ámbito universitario, que, al realizar trabajos en grupo, alguna persona del grupo no asuma su papel de manera responsable. Con la información obtenida a través de esta autoevaluación, se aprecia que el debate, el reparto equitativo del trabajo y asumir el rol asignado son los aspectos peor valorados por el alumnado, lo que lleva a pensar que, a pesar de los beneficios que aporta aprendizaje cooperativo en el trabajo en grupo, es necesario continuar trabajando las habilidades del alumnado para trabajar en grupo de manera cooperativa, eficiente y satisfactoria.

Así pues, tomando en consideración los resultados obtenidos en esta investigación, cabe destacar el elevado grado de aceptación del aprendizaje cooperativo, como medio de aprendizaje por parte de nuestro alumnado universitario de educación física. Este hecho ha de motivar al profesorado universitario de educación física a llevar a cabo experiencias docentes de esta índole y a iniciar estudios sobre el grado de aceptación del alumnado.

\section{Limitaciones}

El presente estudio tiene algunas limitaciones. En primer lugar, el alumnado puede tener una tendencia de respuesta al alza al tener que valorar la tarea docente del profesorado universitario que, posteriormente, deberá evaluar la tarea del alumnado.

En segundo lugar, a la hora de tener una representatividad equitativa entre los dos sexos nos encontramos con una mayor presencia del sexo femenino en el grado de primaria de segundo curso de la Universidad de las Islas Baleares. Este aspecto, enfocado desde la perspectiva de género nos podría dar indicios que esta metodología de trabajo conseguiría acercar la educación física de forma positiva al sector femenino.

En tercer lugar, la variación de componentes en los grupos de trabajo puede desvirtuar los resultados obtenidos, ya que la organización general y la distribución de roles varia.

Finalmente, el proceso de introducción de esta metodología es progresivo. Se han detectado, todavía, algunos grupos que entienden el trabajo cooperativo como una repartición de tareas sin roles, sin reuniones ni revisiones globales, hecho que puede desvirtuar ligeramente el estudio.

\section{Futuras líneas de investigación}

El presente estudio cuenta con una muestra considerable de estudiantes, con medidas repetidas de las variables analizadas y con una muestra con características homogéneas respecto al perfil de estudiante universitario, por lo que se podrán realizar comparativas interesantes con otros estudios sobre esta población. Del mismo modo, se podría incorporar que no había diferencias entre grupos en cuanto al sexo se refiere. Así, se podría realizar un análisis en perspectiva de género y observar si la utilización de esta metodología consigue que este sector valore y disfrute más la educación física.

Al tener continuidad el profesorado con esta asignatura, permitirá continuar con el trabajo presentado, haciendo modificaciones necesarias para mejorar el proceso de enseñanza-aprendizaje a través del trabajo cooperativo. Además, se implementará en otras asignaturas del resto de cursos del grado de educación primaria para poder realizar un seguimiento del alumnado a lo largo de los cuatros cursos del grado.

Por último, futuras investigaciones se centrarán en un análisis del grado de aceptación del alumnado respecto de la utilización del aprendizaje cooperativo, combinado metodologías de análisis de resultados cuantitativas y cualitativas, para analizar en mayor profundidad las preguntas abiertas del diario de prácticas.

\section{Conclusiones}

De acuerdo con lo expuesto anteriormente, concluimos que, en un grupo de alumnos y alumnas del grado de educación primaria, la corresponsabilidad en el proceso de enseñanza-aprendizaje aumenta la motivación del alumnado y, por ende, los resultados en cuanto a autoevaluación, eva- 
luación de la asignatura y la tarea docente. Ratificamos la importancia de la interacción alumnado-profesorado, la cual implica, una profunda cooperación y comunicación entre alumnado y profesorado. La utilización del aprendizaje cooperativo como medio que permite integrar, crear, y compartir conocimientos, facilita el aprendizaje del alumnado.

Además, el aprendizaje cooperativo en la Universidad se erige como una metodología participativa e innovadora, que da continuidad a etapas precedentes (infantil, primaria y secundaria) y que cada vez se hace más fuerte en el mundo educativo. Pese a que las experiencias del uso del aprendizaje cooperativos son cada vez más frecuentes en determinadas titulaciones especialmente vinculadas a educación, aún queda un largo camino a recorrer dentro lo que es la enseñanza universitaria sin distinción de ramas de conocimiento. Por ello, seguir investigando en metodologías cooperativas, ayudando a la formación de calidad debe ser una prioridad, pues permitirá formar a profesionales maestros y maestras con capacidad de reflexión, innovadores y con una base pedagógica crítica. Destacar que el presente estudio es un estudio piloto, con el que se pretende seguir trabajando en la investigación del trabajo cooperativo como metodología de aprendizaje, además de como contenido a enseñar. Para finalizar, este estudio, vinculado con las aportaciones del trabajo de Pérez Pueyo y Hortigüela Alcalá (2019), ha permitido observar que la metodología del aprendizaje cooperativo es efectiva demostrando un impacto positivo y tangible de mejora en las prácticas del alumnado, sostenible por ser capaz de mantenerse en el tiempo, y replicable en diferentes contextos universitarios e incluso de enseñanza obligatoria.

\section{Agradecimientos}

Los autores agradecen a los estudiantes su participación, y al Instituto de Investigación e Innovación Educativa (IRIE), de la Universitat de les Illes Balears su financiación. Así como a otros profesionales de la Universitat de les Illes Balears por su colaboración en el presente proyecto.

\section{Financiación}

Convocatoria de ayudas para la innovación y la mejora de la calidad docente. PID:181920. Instituto de Investigación e Innovación Educativa (IRIE), Universitat de les Illes Balears, España.

\section{Referencias}

Armada, J. M., Gonzailez, I., y Montaìvez, M. (2013). La expresioìn corporal: un proyecto para la inclusioìn. Retos: Nuevas Tendencias en Educacioìn Fì̀sica, Deporte y Recreacioìn, 24, 107-112.

Bayraktar, G. (2011). The effect of cooperative learning on students' approach to general gymnastics course and academic achievements. Educational Research and Reviews, 6(1), 62-71.

Casey, A., y Goodyear, V. A. (2015). Can Cooperative learning achieve the four learning outcomes of physical education? A review of literature. Quest, 67(1), 56-72.

Cherng, H., Turney, K., y Kao, G. (2014). Less socially engaged? Participation in friendship and extracurricular activities among racial/ethnic minority and immigrant adolescents. Teachers College Record, 116(3), 1-28.

Chickering, A. y Gamson, Z. (1991). Applying the seven principies for good practice in undergraduate education. New directions for Teaching and Leaming, Documento 47. George Masón University. Crespo, R. (2014). Bailes tradicionales escoceses como actividad física cooperativa en la Educación Primaria y Secundaria. En C. Velázquez, J. Roanes y F. Vaquero (coords.) Actas del IX Congreso Internacional de Actividades Físicas Cooperativas (pp. 468-475). Valladolid: La Peonza.

Cueto, D. (2014). El trabajo mediante tarjetas en Educación Física: cooperación y variedad en las actividades de orientación deportiva para el Tercer Ciclo de Educación Primaria. En C. Velázquez, J. Roanes y y F. Vaquero (coords.) Actas del IX Congreso Internacional de Actividades Físicas Cooperativas (pp. 338-350). Valladolid: La Peonza.

Feria-Madueño, A., Grimaldi-Puyana, M., Sánchez-Oliver, A. J., y Alcaraz-Rodríguez, V. (2017). Aprendizaje cooperativo y su percepción en futuros docentes de educación física. TRANCES. Transmisión del Conocimiento Educativo y de la Salud, 1, 189-198.

Fernández del Río, J. (2003): El aprendizaje cooperativo en el aula de educación física. Análisis comparativo con otros sistemas de enseñanza y aprendizaje. La Peonza Publicaciones. Valladolid. Cederrón.

Fernández-Río, J. (2010). La enseñanza de las habilidades motrices básicas a través de estructuras de trabajo cooperativas. En C. Velázquez (coord.) Aprendizaje Cooperativo en Educación Física. Fundamentos y aplicaciones prácticas (pp. 165-186). Barcelona: Inde.

Fernández-Río, J. (2014). Aportaciones del modelo de responsabilidad personal y social al aprendizaje cooperativo. In Actas del IX Congreso Internacional de Actividades Físicas Cooperativas (pp. 18-32).

Fernández-Río, J. (2017). El Ciclo del Aprendizaje Cooperativo: una guía para implementar de manera efectiva el aprendizaje cooperativo en educación física. Retos: nuevas tendencias en educación física, deporte y recreación, 32, 264-269.

Fernández-Río, J. y Méndez-Giménez, A. (2013). El aprendizaje cooperativo como marco metodológico para la enseñanza de las habilidades gimnásticas en el ámbito educativo. Revista Española de Educación Física y Deportes, 400, 38-53.

Fernández-Río, J. y Méndez-Giménez, A. (2016). El aprendizaje cooperativo: Modelo pedagógico para Educación Física. Retos. Nuevas Tendencias en Educación Física, Deporte y Recreación, 29, 201-206.

Gibbons, S., y Black, K. M. (1997). Effect of participation in team building activities on the self-concepts of middle school physical education students. Avante, 1, 46-60.

Gillies, R. M. (2014). Developments in Cooperative Learning: Review of Research. Anales de Psicología,, 30(3), 792-801.

Hannon, J. C. y Ratliffe, T. (2004). Cooperative learning in physical education. Strategies, 17(5), 29-32.

Hernández, A. (2018). Gestión de grupos de aprendizaje cooperativos en el ámbito universitario. XI Congreso Internacional de Actividades Físicas Cooperativas. Universidad de Oviedo, Avilés.

Herrada, R. I. y Baños, R. (2018). Revisión de experiencias de aprendizaje cooperativo en ciencias experimentales. Campo Abierto. Revista de Educación, 37(2), 157-170.

Izquierdo, T., Asensio, E., Escarbajal, A. y Rodriìguez, J. (2019). El aprendizaje cooperativo en la formacioìn de maestros de Educacioìn Primaria. Revista de Investigacioin Educativa, 37(2), 543-559. DOI: http://dx.doi.org/10.6018/rie.37.2.369731

Johnson, D. y Johnson, R. (1999). Aprender juntos y solos. Aprendizaje cooperativo, competitivo e individualista. Buenos Aires: Aique.

Johnson, D. y Johnson, R. (2014). Cooperative learning in 21st Century. Anales de Psicologiìa, 30(3), 841-851.

Lamoneda, J., González-Víllora, S. y Fernández-Río, J. (2020). Hibridando el Aprendizaje Cooperativo, la Educación Aventura y la Gamificación a través de la carrera de orientación. Retos. Nuevas Tendencias en Educación Física, Deporte y Recreación, (38), 754-760.

Latorre-Cosculluela, C., Liesa-Orús, M. y Vázquez-Toledo, S. (2018). Escuelas inclusivas: aprendizaje cooperativo y TAC con alumnado con TDAH. Magis, Revista Internacional de Investigación en Educación, 10(21), 137-152. 
Lavega, P., Planas, A. y Ruiz, P. (2014). Juegos cooperativos e inclusioìn en Educacioìn Fiìsica. Revista Internacional de Medicina y Ciencias de la Actividad Fiìsica y el Deporte, 14(53), 3751.

Legrain, P., Escalié, G., Lafont, L. y Chaliès, S. (2019). Cooperative learning: a relevant instructional model for physical education pre-service teacher training? Physical Education and Sport Pedagogy, 24(1), 73-86.

Maldonado, M. (2007). El trabajo colaborativo en el aula universitaria. Laurus, 13(23), 263-278.

Martín, G. (2014). Utilización del aprendizaje cooperativo para la transformación de los aprendizajes del alumnado y la formación continua de las maestras en un centro rural agrupado (Tesis Doctoral). Universidad de Valladolid, Valladolid.

Naranjo, M. y Lago, J.R. (2017). Aprenentatge cooperatiu a l'ensenyament universitari: el programa «Cooperar per Aprendre. Aprendre a Cooperar. Universitat de Vic.

Navarro-Patón, R. N., Rego, B. y García, M. (2018). Incidencia de los juegos cooperativos en el autoconcepto físico de escolares de educación primaria. Retos. Nuevas Tendencias en Educación Física, Deporte y Recreación, 34, 14-18.

Pastor, V. M. L., Brunicardi, D. P., Arribas, J. C. M. y Aguado, R. M (2016). Los retos de la Educación Física en el Siglo XXI. Retos. Nuevas tendencias en Educación Física, deporte y recreación 29, 182-187.

Pelaz, D. (2018). Aprendizaje cooperativo en un aula de Educación Primaria: un estudio de caso (Tesis de maestría). Universidad de Valladolid, Valladolid.

Pérez Pueyo, Á. y Hortigüela Alcalá, D. (2019). ¿Y si toda la innovación no es positiva en Educación Física? Reflexiones y consideraciones prácticas. Retos. Nuevas Tendencias en Educación Física, Deporte y Recreación, 37, 579-587.

Prieto, J. M. (2018). Gamificación del aprendizaje y motivación en universitarios. Elaboración de una historia interactiva: MOTORIA-X. Edutec. Revista Electrónica de Tecnología Educativa, 66, 77-92.

Pujolàs, P. (2008a). El aprendizaje cooperativo como recurso y como contenido. Aula de innovación educativa, 170, 37-41.

Pujolás, P. (2008b). 9 ideas clave. El aprendizaje cooperativo. Barcelona: Graó.

Pujolàs, P., Lago, J. R., Naranjo, M., Pedragosa, O., Riera, G., Soldevila, J., Olmos, G., Torner, A. y Rodrigo, C. (2014). El programa CA/ AC (Cooperar para Aprender/Aprender a Cooperar) para enseñar a aprender en equipo Implementación del aprendizaje cooperativo en el aula. Retrieved from https://www.elizalde.eus/wpcontent/uploads/izapideak/CA-ACprograma.pdf

Ramos, M. A. y Hernangómez, A. (2014). Actividades rítmicas (batuka y zumba) aplicadas al ámbito educativo de primaria y/o secundaria mediante metodología cooperativa. En C. Velázquez, J. Roanes y F. Vaquero (coords.) Actas del IX Congreso Internacional de Actividades Físicas Cooperativas (pp. 468-475). Valladolid: La Peonza.

Velázquez, C., Fraile, A. y López-Pastor, V.M. (2014). Aprendizaje cooperativo en Educación Física. Movimento: revista da Escola de Educação Física, 20(1), 239-259.

Velázquez, C. (2015). Aprendizaje cooperativo en Educación Física: estado de la cuestión y propuesta de intervención. Retos. Nuevas Tendencias en Educación Física, Deporte y Recreación, 28,
234-239.

Woods, D. M. y Chen, K.-C. (2011). Evaluation Techniques For Cooperative Learning. International Journal of Management \& Information Systems, 14(1), 1-6. https://doi.org/10.19030/ ijmis.v14i1.815

Anexo 1.

Preguntas del diario de prácticas realizado a través de la metodología de trabajo cooperativo.

1. ¿Que hemos aprendido?

2. ¿Cómo nos hemos sentido durante la sesión?

3. ¿Cómo podemos relacionar la sesión con los contenidos teóricos explicados en el aula?

Anexo 2.

Evaluación de la sesión, apartado incluido en el diario de prácticas. EVALUACIÓN SESIÓN Valora del 1-10

\begin{tabular}{|c|c|c|c|c|c|c|c|c|c|c|c|}
\hline & 1 & 2 & 3 & 4 & 5 & & & 7 & 8 & 9 & 10 \\
\hline \multicolumn{12}{|l|}{ Bienestar } \\
\hline \multicolumn{12}{|l|}{ Grado de participación } \\
\hline \multicolumn{12}{|l|}{\begin{tabular}{|l|} 
Clima de clase \\
\end{tabular}} \\
\hline \multicolumn{12}{|l|}{ Comunicación P-A A-PA-A } \\
\hline \multicolumn{12}{|l|}{ Consecución de objetivos } \\
\hline & 1 & 2 & 3 & 4 & & & 6 & 7 & 8 & 9 & 10 \\
\hline \multicolumn{12}{|l|}{ Trabajo individual } \\
\hline Trabajo de tus compañeros/as de grupo & & & & & & & & & & & \\
\hline Trabajo del profesor/a & & & & & & & & & & & \\
\hline
\end{tabular}

Anexo 3.

Diseño de actividades relacionadas con la sesión llevada a la práctica, apartado incluido en el diario de prácticas.

Prepara:

-Dos actividades trabajando la discriminación sensorial-visual para primer ciclo de educación primaria.

-Dos actividades trabajando la discriminación táctil para segundo ciclo de educación primaria -Una actividad de vuelta a la calma relacionada con los contenidos de la sesión (Discriminación sensorial)

\section{Anexo 4.}

Valoración del trabajo cooperativo por grupos de trabajo. \begin{tabular}{|l|l|l|l|l}
\hline Todo el grupo ha ejercido, durante la realización del diario de prácticas, las & 1 & 2 & 3 & 4
\end{tabular} func el grupo ha ejercido, \begin{tabular}{l} 
funciones propias de su rol. \\
$\begin{array}{l}\text { Todo el grupo ha realizado las tareas que se habían comprometido a } \\
\text { realizar. }\end{array}$ \\
\hline
\end{tabular}

realizar.

\begin{tabular}{|l|l|l|l|l|}
\hline Todo el grupo ha asistido a las reuniones de trabajo. & 1 & 2 & 3 & 4 \\
\hline Todo el grupo ha participado de forma equitajiva durante las sesiones de & 1 & 2 & 3 & 4 \\
\hline
\end{tabular} \begin{tabular}{|l|l|l|l|l|l|l|l|l|}
\hline Todo el grupo ha participado de forma equitativa durante las sesiones de & 1 & 2 & 3 & 4 \\
\hline
\end{tabular} \begin{tabular}{|l|l|l|l|l|l|l|l|l|l|}
\hline Las decisiones se han tomado de forma consensuada entre todo el grupo de & 1 & 2 & 3 & 4 \\
\hline
\end{tabular} \begin{tabular}{|l|l|l|l|l|l|}
\hline Ha habido debate, discusión y contraste de opiniones durante las reuniones & 1 & 2 & 3 & 4 \\
\hline
\end{tabular} $1=$ Nada o muy poco $2=$ un poco $3=$ Bastante $4=$ Mucho

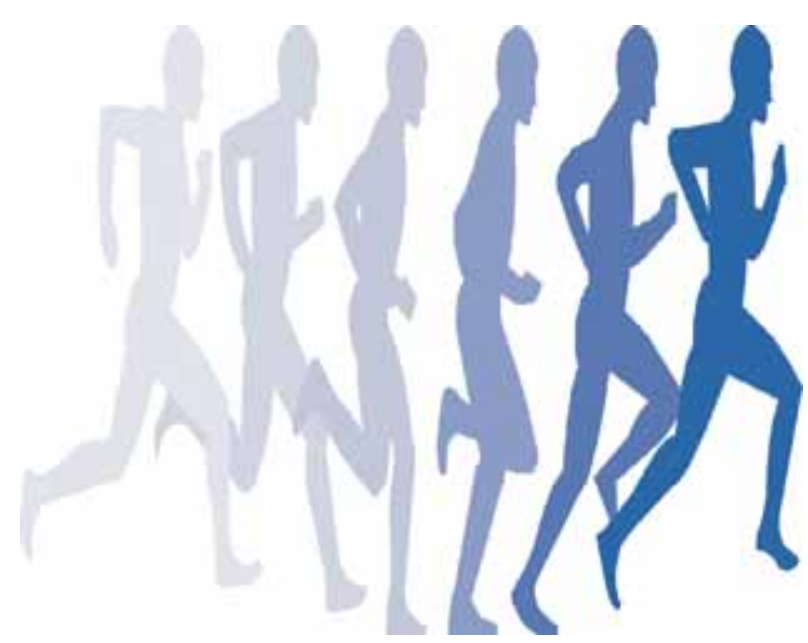
Anexo 5 .

Rúbrica para la evaluación del diario de prácticas.

\begin{tabular}{|c|c|c|c|c|}
\hline \multicolumn{4}{|c|}{ Rúbrica evaluación diario de prácticas } & \multirow[t]{2}{*}{ Grupo: } \\
\hline & Insuficiente & $\begin{array}{l}\text { Suficiente } \\
\end{array}$ & Notable & \\
\hline $\begin{array}{l}\text { 1.Redacción, coherencia y } \\
\text { ortografia }\end{array}$ & $\begin{array}{l}\text { El diario tiene una gran cantidad } \\
\text { de faltas de ortografia y errores } \\
\text { graves de coherencia. }\end{array}$ & $\begin{array}{l}\text { El diario tiene algunas faltas de } \\
\text { ortografía no significativas con } \\
\text { algunos errores de coherencia. }\end{array}$ & $\begin{array}{l}\text { El diario no tiene faltas de ortografia ni } \\
\text { errores de coherencia. La redacción es } \\
\text { clara y fácilmente legible. }\end{array}$ & $\begin{array}{l}\text { i El diario no tiene faltas de ortografia ni errores de } \\
\text { coherencia. La información es clara, accesible y concreta. }\end{array}$ \\
\hline $\begin{array}{l}\text { 2. Grado de reflexión sobre } \\
\text { las sesiones }\end{array}$ & $\begin{array}{l}\text { El documento no presenta } \\
\text { reflexiones, sino simplemente } \\
\text { detalla las actividades realizadas. }\end{array}$ & $\begin{array}{l}\text { El documento presenta las } \\
\text { actividades con algunas } \\
\text { reflexiones sobre la práctica. }\end{array}$ & $\begin{array}{l}\text { El documento es reflexivo, con un } \\
\text { análisis crítico y presenta una línea } \\
\text { común entre el grupo. }\end{array}$ & $\begin{array}{l}\text { El documento es totalmente reflexión. Es fácilmente } \\
\text { perceptible una puesta en común entre todo el grupo. }\end{array}$ \\
\hline $\begin{array}{l}\text { 3. Grado de adecuación de las } \\
\text { actividades propuestas }\end{array}$ & $\begin{array}{l}\text { Las actividades no son adecuadas } \\
\text { los objetivos o contenidos } \\
\text { propuestos. }\end{array}$ & $\begin{array}{l}\text { aAlgunas de las actividades no son } \\
\text { adecuadas a los objetivos o } \\
\text { contenidos propuestos. }\end{array}$ & $\begin{array}{l}\text { La mayoría de las actividades cumplen } \\
\text { los objetivos o contenidos propuestos. }\end{array}$ & $\begin{array}{l}\text { El total de las actividades se ajustan a los objetivos y } \\
\text { contenidos propuestos. }\end{array}$ \\
\hline $\begin{array}{l}\text { 4. Valoración final y } \\
\text { bibliografía }\end{array}$ & $\begin{array}{l}\text { El documento no contiene } \\
\text { valoración final ni/o bibliografia. }\end{array}$ & $\begin{array}{l}\text { El documento presenta una } \\
\text { valoración final sencilla, poco } \\
\text { reflexiva con/sin bibliografia. }\end{array}$ & $\begin{array}{l}\text { El documento resume con detalle las } \\
\text { experiencias del grupo. Incluye } \\
\text { bibliografia. }\end{array}$ & $\begin{array}{l}\text { El documento presenta una línea común de razonamiento } \\
\text { con detalles sobre las experiencias del grupo. Incluye } \\
\text { bibliografía correctamente referenciada. }\end{array}$ \\
\hline $\begin{array}{l}\text { 5. Participación de los } \\
\text { componentes }\end{array}$ & $\begin{array}{l}\text { No ha habido participación de } \\
\text { todos los miembros del grupo. }\end{array}$ & $\begin{array}{l}\text { Ha habido participación de todos } \\
\text { los miembros del grupo, pero no } \\
\text { de forma equitativa. }\end{array}$ & $\begin{array}{l}\text { Ha habido participación equitativa de } \\
\text { todo el grupo. }\end{array}$ & $\begin{array}{l}\text { Ha habido participación equitativa de todas las personas } \\
\text { del grupo, y se percibe claramente a lo largo del } \\
\text { documento. }\end{array}$ \\
\hline
\end{tabular}

